entreprises par le Centre en collaboration avec plusieurs professeurs de l'Université d'Élisabethville:

I. L'étude ethnologique du Haut Katanga (géographie ethnologique et mouvement des populations depuis la conquête, acculturation), par Mmes Lebeuf et Pouleur.

2. L'étude de sociologie urbaine sur la population du nouveau quartier extra-coutumier de l'Arboretum à Ruashi: développement de cette communauté; caractéristiques de sa population; l'influence du milieu traditionnel; facteurs et conditions de productivité dans les entreprises; niveau de vie et budgets familiaux; professions et salaires; organisation sociale.

3. L'étude des méthodes d'enquête statistique etc. concernant l'absentéisme et l'instabilité des travailleurs sera dirigée par M. Glume, Professeur de mathématiques à l'Université d'Élisabethville.

4. M. Bietlot, professeur de l'Université, y poursuivra des recherches sur l'évolution des concepts du milieu coutumier en milieu extra-coutumier.

5. M. Colette, Chargé des cours de psychologie, s'intéressera à la mise en place d'un service d'orientation scolaire.

6. M. Polome, Professeur de linguistique, dirigera, au Centre, des recherches sur les modifications du kingwana local sous l'influence des langues vernaculaires et du nouveau parler.

7. M. le Professeur Hiernaux, spécialiste en anthropologie physique, se préoccupe d'une enquête sur la croissance, portant tant sur des groupes de sujets d'âge connu que sur des enfants suivis dès leur naissance ainsi que d'une étude sur les jumeaux monozygotes.

8. M. Van Roye, spécialiste de questions économiques, sera incessamment attaché au Centre pour y poursuivre des recherches sur l'économie du Katanga.

Au quartier extra-coutumier de l'Atboretum à Ruashi ont été établis un foyer social et un centre éducatif et social dont l'Institut Solvay a la gestion. A un foyer social rural à Bongandanga, Province d'Équateur, d'intéressantes méthodes de promotion du milieu indigène en zone rural seront appliquées; l'action de ce foyer rural sera menée sur la base des observations ethnologiques de M. Luc de Heusch de l'Université de Bruxelles qui passera un an dans la région.

En outre a été confiée à l'Institut Solvay la gestion de l'Institut Supérieur d'Études sociales à Élisabethville chargé de la formation des travailleurs sociaux tant européens qu'indigènes.

\title{
Inauguration d'un Laboratoire de l'I.R.S.A.C.
}

DE nombreuses personnalités métropolitaines et locales, parmi lesquelles MM. de Vleeschauwer, ancien ministre des Colonies, Gillet, gouverneur de la Société Générale, Ryckmans, gouverneur général honoraire, Martin, gouverneur de la Banque centrale, et Willems, vice-président de la Fondation universitaire, ont assisté à Élisabethville à l'inauguration du laboratoire de l'Institut pour la recherche scientifique en Afrique centrale (Irsac).

M. De Bruyne, président du Conseil d'administration de l'Irsac, a rappelé que l'Institut avait inauguré au cours des dernières semaines les centres de Mabali, en pleine forêt équatoriale sur le lac Tumba, et de Lwiro. L'Institut, a-t-il poursuivi, fondé en 1947 a ouvert l'année suivante les centres d'Uvira et d'Astrida, le premier plus spécialement consacré aux sciences de la nature, le second aux problèmes humains. Devant les initiatives et les efforts de personnalités katangaises, qui fondèrent notamment le Centre d'étude des problèmes scciaux indigènes, l'Irsac a décidé d'installer un centre à Élisabethville, consacré plus spécialement aux études de la parasitologie et des problèmes posés dans les miliex ruraux par les populations indigènes. 
Le docteur Boneel, directeur du centre d'Élisabethville, a donné ensuite des explications sur les laboratoires, qui poursuivront des études poussées sur les trypanosomes vecteurs, notamment la maladie du sommeil. L'équipement des laboratoires est ultramoderne, màis les chercheurs manquent, a-t-il déclaré. Le docteur Boneel a adressé alors un appel aux universitaires belges pour signaler les carrières qui existent au Congo belge.

\section{Un Congolais nommé Attaché de Cabinet au Ministère des Colonies}

M. Buisseret, ministre des Colonies, a décidé d'attacher à son cabinet M. Antoine-Roger Bolamba, écrivain et journaliste congolais. M. Bolamba est rédacteur en chef de La Voix du Congolais depuis sa fondation en r945. Avant cette date, il fut pendant douze ans attaché au secrétariat du médecin-directeur du Foréami. M. Bolamba est détenteur de la carte du mérite civique, membre du Comité consultatif de l'Office des Cités Africaines, co-président du groupement culturel belgo-congolais, membre du Comité consultatif de Radio Congo belge - Émissions africaines, et membre du Comité des Amis de l'Art indigène.

\section{Les Problemes de l'Enseignement secondaire (A.O.F.)}

M. JEAN CAPELle, recteur de l'Université d'A.O.F., vient d'effectuer à Conakry un court séjour au cours duquel, en liaison avec les services compétents, il a étudié plus particulièrement les problèmes de la réorganisation de l'enseignement secondaire et technique.

Dans un double souci d'efficacité et d'économie et afin de permettre aux élèves de se spécialiser à partir de la classe de quatrième seulement après avoir reçu un enseignement commun dans les classes précédentes, la Direction générale de l'Enseignement envisage, en effet, pour chaque territoire, de concentrer en un établissement unique ou ' collège d'orientation mixte', toutes les classes de sixième et de cinquième de l'enseignement secondaire, qu'il s'agisse du classique, du moderne ou du technique.

Quant aux classes allant de la quatrième à la deuxième partie du baccalauréat, elles seraient également concentrées dans un établissement unique, un ' lycée ou collège mixte' classique, moderne et technique, les élèves ayant choisi l'une des trois branches à sortie du collège d'orientation, selon leurs goûts et leurs capacités et les conseils de leurs professeurs.

Cette formule, qui constitue un regroupement horizontal des différentes classes de l'enseignement secondaire actuellement réparties entre plusieurs établissements - collège technique, collège moderne, collège classique - est déjà appliquée avec succès à Abidjan. Elle est projetée à Dakar, mais les difficultés d'installation n'ont pas encore permis de l'organiser.

A Conakry, où se poursuivent les grands travaux d'aménagement de bâtiments scolaires, elle serait susceptible d'intervenir à la rentrée scolaire d'octobre 1957.

Pendant son séjour à Conakry, le recteur Capelle s'est également penché sur le problème de la formation professionnelle et a pris contact à ce sujet avec les dirigeants des deux grandes sociétés industrielles de la place: la Compagnie des Bauxites du Midi et la Compagnie Minière de Conakry.

\section{Institut Français d'Afrique Noire}

LA Section de Sociologie de l'IFAN, réduit temporairement à trois membres depuis le départ de P. Mercier, poursuit son étude de problèmes posés par l'urbanisation et l'industrialisation de l'Ouest Africain.

A. Hauser effectue depuis I953 une enquête de sociologie industrielle dans l'Ouest Africain en procédant par interview des principaux agents européens et travailleurs africains d'entreprises diverses. La main-d'œuvre a été décrite quantitativement dans la mesure où 